\title{
Congestion Pricing Design based on Gini Coefficient under the Combined Travel
}

\author{
Chaohui Luo ${ }^{1, a}$, Dalong Mo 2, b, * \\ Mathematics and Computer Information Engineering Department, Baise University, Guangxi, \\ 533000, China \\ Hezhou University Faculty of Science, Guangxi Hezhou 542899, China \\ alchaohui@21cn.com, ’lchaohui@163.com
}

Keywords: Urban Transportation; Combined Travel; congestion pricing; Gini Coefficient; Park and Ride; Two-layer Model.

\begin{abstract}
Considering three travel modes of taking a bus, self-driving and park-and-ride, it studies the congestion pricing under the combined travel. While designing the congestion pricing, it considers the fairness, takes road resources occupied by each mode as the fairness indicator, transforms the traditional upper model by Gini Coefficient, constructs an upper model with little total cost of traffic network and Gini Coefficient as much as possible and gives constraint conditions of Gini Coefficient. The example shows that the congestion pricing design based on Gini Coefficient can improve the traffic congestion of roads while taking fairness into consideration.
\end{abstract}

\section{Introduction}

Combined travel, refers to the transfer of several transportation means to arrive at the destination. In the face of growing traffic congestion, many cities encourage people to use high-capacity public transport or combined travel to reduce vehicle travel, so as to alleviate traffic congestion. Therefore, a lot of study on combined travel has been made by many scholars ${ }^{[1-5]}$. Meng Meng ${ }^{[1]}$ studied the distribution of traffic flow on mixed traffic road network, considering the bicycle rental policy and park-and-ride situation. Huang Haijun and Li Zhichun ${ }^{[3,4]}$ focused on the combined travel of park-and-ride under fixed and elastic demand. Li Honglian ${ }^{[5]}$ described a traffic network model consisting of 4 transportation means using super network and proposed the SUE Model under multi-mode transport network condition. The above scholars have made comprehensive studies on the decision behaviors of combined travel. In order to alleviate traffic congestion, based on the previous study on combined travel, discussions on parking charge and congestion charge are thus considered. With reasonable pricing, encourage travelers to change their ways to travel and vehicles on road, and finally to alleviate traffic congestion.

Shi Feng, Li Hengxin and Wang Jian ${ }^{[6-9]}$ had made research on the comprehensive optimization of the two charge strategies, namely parking and congestion charge. The two-layer model also reflects the game behavior between charge administrators and travelers, with the upper layer reflecting the decision and goal of administrators while the lower layer describing travelers' travel behaviors. However, the above research lacked consideration of social equity. As transportation infrastructure, roads are public goods with strong social welfare. Given that different travelling means' occupation on road resources varies, when determining charge fees, a question must be raised, that whether the measure has provided different travel groups with equal travelling accessibility or improved accessibility in aspects of resource occupation, travel target and user efficiency, etc. ${ }^{[10]}$.

Some scholars studied traffic fairness with Lorenz curves and Gini coefficient ${ }^{[10-14]}$. Urban road resources occupied by each travel mode as measurable indicator, using Lorenz curve and the Gini coefficient, Juan Zhicai ${ }^{[10]}$ pointed out the influence of congestion pricing on the fairness of road resources occupation by different travel modes.Using Gini coefficient, Li Yishun ${ }^{[14]}$ evaluated the fairness of the implementation of regional congestion charge and park-and-ride combination. 
However, the studies are only quantitative evaluation of traffic fairness by Gini coefficient, with few application of Gini coefficient in congestion charge design.

Based on the previous studies many scholars, this paper studies the congestion charge in combined travel considering traffic fairness. Using Gini coefficient to transform traditional upper model and joining Gini coefficient function, construct a function with low total network travel cost and small Gini coefficient as possible, and set the constraint of Gini coefficient in the meantime, to achieve the congestion charge design under different fairness requirements.

\section{Network Description}

This paper studies the travel behavior from the suburb to city. Travelers travel from the suburb R to downtown area $S$ to work, and at the traffic boundary between THE suburb and city is the charging parking spot $\mathrm{P}$. After arriving at the parking spot $\mathrm{P}$, the car drivers must choose to pay congestion fee to drive to the city or pay parking fee to take public transport to city. Generally, the working travelers have comparatively fixed travel demand and will not reduce travelling frequency to city because of the increase of congestion fees or traveling cost but change their travel mode to the city. Therefore, this paper has fixed demand SUE model as lower model and the minimum total network travel cost of upper model as the objective.

Assume i travel modes, $i=1,2,3$, respectively bus travelling the whole travel, driving the whole travel and park-and-ride. Uniting the dimension, this paper takes time as a unified measurement standard, with all the cost for parking, bus travel or congestion converted into time cost. A is the car section set; B is bus section set; $x_{a}^{i}$ is the flow of different modes on Section a; $t_{a}^{i}\left(x_{a}^{i}\right)$ is the travel time of different modes on Section a.

\section{General cost}

\subsection{General cost of bus travel}

Buses cost no congestion charge to reflect the policy of bus priority. The general cost of bus travel includes travel time, waiting time and bus fare. Assume the traveler is charged a fixed fee only once from starting station to destination, thus the general expected cost of bus travel on route $\mathrm{K}$ is:

$$
c_{r s, k}^{1}=\sum_{a \in B} t_{a}^{1}\left(x_{a}^{1}\right) \cdot \delta_{a, k}^{r s}+M_{r s, k}
$$

Wherein $\delta_{a, k}^{r s}$ is correlation coefficient. If Road $a$ is on the Route $\mathrm{K}$ and $(r, s)$ connecting OD, then $\delta_{a, k}^{r s}$ is 1 , otherwise $0 . M_{r s, k}$ Is the integrated time cost converted from bus fare and waiting time on Route K. according to actual situation, $M_{r s, k}$ is generally regarded as a constant.

\subsection{General cost of driving the whole travel}

The general cost of driving the whole travel includes travel time, congestion charge and so on. So the general expected cost of driving the whole travel on road a is:

$$
c_{a}^{2}\left(x_{a}^{2}, u_{a}\right)=t_{a}^{2}\left(x_{a}^{2}\right)+u_{a}
$$

Wherein $u_{a}$ is the congestion charge on Road a, thus the general expected cost of driving the whole travel on Route $\mathrm{K}$ is:

$$
c_{r s, k}^{2}=\sum_{a \in A} c_{a}^{2}\left(x_{a}^{2}, u_{a}\right) \delta_{a, k}^{r s}=\sum_{a \in A}\left[t_{a}^{2}\left(x_{a}^{2}\right)+u_{a}\right] \delta_{a, k}^{r s}
$$

\subsection{General cost of Park-and-ride}

The general cost of combined travel includes travel time by car, parking fees, waiting time, travel time by bus and bus fare. So the whole travel time on Road A is:

$$
t_{a}^{3}\left(x_{a}^{3}\right)=\left\{\begin{array}{l}
t_{a}^{2}\left(x_{a}^{3}\right), a \in A \\
t_{a}^{1}\left(x_{a}^{3}\right), a \in B
\end{array}\right.
$$

Therefore, the general expected cost of park-and-ride on Route $\mathrm{K}$ is: 


$$
c_{r s, k}^{3}=\sum_{a \in A} t_{a}^{2}\left(x_{a}^{3}\right) \delta_{a, k}^{r p}+T_{p}+\sum_{a \in B} t_{a}^{1}\left(x_{a}^{3}\right) \delta_{a, k}^{p s}+M_{p s, k}
$$

Wherein $T_{p}$ is the parking fee at parking spot $p ; M_{p s, k}$ is the integrated time cost converted from bus fare and waiting time after transfer.

The integrated value of the car after the transfer time, etc., bus fare and other expenses are translated into time; wherein the parking fees charged at the point of transfer.

\section{Lower SUE model of fixed demand}

The lower SUE model of fixed demand describes the traveler's choice of travel mode and route. According to Reference ${ }^{[15]}$, the lower SUE model of fixed demand P1 describes the traveler's behavior as:

$$
\begin{aligned}
& \text { (P1) } \min Z\left(\boldsymbol{x}^{i}, \boldsymbol{f}^{i}, \boldsymbol{q}^{i}\right)=\sum_{i=1}^{3}\left[\frac{1}{\theta} \sum_{r s} \sum_{k} \int_{0}^{f_{r s, k}^{i}} \ln w d w-\left(\frac{1}{\theta}-\frac{1}{\beta}\right) \sum_{r s} \int_{0}^{q_{r s}^{i}} \ln w d w-\sum_{r s} h_{r s}^{i} q_{r s}^{i}\right] \\
& +\left[\sum_{a \in B} \int_{0}^{x_{a}^{1}} t_{a}^{1}(w) d w+\sum_{k} \sum_{r s} f_{r s, k}^{1} M_{r s, k}\right]+\sum_{a \in A} \int_{0}^{x_{a}^{2}} c_{a}^{2}\left(w, u_{a}\right) d w \\
& +\left[\sum_{a \in A} \int_{0}^{x_{a}^{3}} t_{a}^{2}(w) d w+\sum_{k} \sum_{r p} f_{r p, k}^{3} T_{p}+\sum_{a \in B} \int_{0}^{x_{a}^{3}} t_{a}^{1}(w) d w+\sum_{k} \sum_{p s} f_{p s, k}^{3} M_{p s, k}\right] \\
& \text { s.t. } \sum_{k} f_{r s, k}^{i}=q_{r s}^{i}, \forall r, s, i \\
& \sum_{i} q_{r s}^{i}=q_{r s}, \forall r, s \\
& x_{a}^{i}=\sum_{r s} \sum_{k} f_{r s, k}^{i} \delta_{a . k}^{r s}, \forall a, i \\
& f_{r s, k}^{i} \geq 0, x_{a}^{i} \geq 0, q_{r s}^{i} \geq 0, q_{r s} \geq 0, \forall r, s, i, k, a
\end{aligned}
$$

Wherein $f_{r s, k}^{i}$ is the traffic flow on Route $\mathrm{K}(r, s)$ to OD using Mode $i ; \boldsymbol{q}_{r s}=\left(\cdots, q_{r s}^{i}, \cdots\right)$ is the traveler' traffic demand in $(r, s)$ to OD; $h_{r s}^{i}$ is the attractiveness of various travel modes. According to the proof in Reference ${ }^{[15]}$, Model P1's first-order condition at local extreme point is equivalent to route selection and mode selection conditions:

$$
\begin{aligned}
& p_{r s, k}^{i}=\frac{\exp \left(-\theta c_{r s, k}^{i}\right)}{\sum_{k} \exp \left(-\theta c_{r s, k}^{i}\right)} \\
& p_{r s}^{i}=\frac{\exp \left[\beta\left(h_{r s}^{i}-S_{r s}^{i}\right)\right]}{\sum_{i} \exp \left[\beta\left(h_{r s}^{i}-S_{r s}^{i}\right)\right]}
\end{aligned}
$$

Wherein $p_{r s, k}^{i}$ is the probability of selecting Route $\mathrm{K}$ using Mode $i ; \theta$ is the traveler' route familiarity, the larger $\theta$ is, the more familiar the traveler is. $p_{r s}^{i}$ is the probability of using Mode $i, \beta$ is selection parameter of travel mode, $h_{r s}^{i}$ is the attractiveness of Mode $i, S_{r s}^{i}\left(c_{r s, k}^{i}\right)$ is the expected cost of Mode $i$ on $(r, s)$ to OD, and ${ }^{[15]}$

$$
S_{r s}^{i}\left(c_{r s, k}^{i}\right)=E\left[\min _{k \in K_{r s}}\left\{C_{r s, k}^{i}\right\}\right]=-\frac{1}{\theta} \ln \sum_{k} \exp \left(-\theta c_{r s, k}^{i}\right)
$$

Therefore Mode P1 can describe the traveler's choice of travel mode and route.

\section{Upper model based on Gini coefficient}

For lower SUE model of fixed demand, the traditional upper model generally pursue the minimum total network travel cost $\mathrm{P} 2$, 


$$
\begin{aligned}
& \text { (P2) } \min _{u} \mathrm{Z}(\boldsymbol{u}, \boldsymbol{x})=\sum_{a \in B} x_{a}^{1} \cdot t_{a}^{1}\left(x_{a}^{1}\right)+\sum_{k} \sum_{r s} f_{r s, k}^{1} M_{r s, k}+\sum_{a \in A} x_{a}^{2} \cdot\left[t_{a}^{2}\left(x_{a}^{2}\right)+u_{a}\right] \\
& +\sum_{a \in A} x_{a}^{3} t_{a}^{2}\left(x_{a}^{3}\right)+\sum_{a \in B} x_{a}^{3} t_{a}^{1}\left(x_{a}^{3}\right)+\sum_{k} \sum_{p s} f_{p s, k}^{3}\left(T_{p}+M_{p s, k}\right) \\
& \text { s.t. } u_{a}^{\min } \leq u_{a}^{i} \leq u_{a}^{\max }, \forall i, a
\end{aligned}
$$

Wherein $\left[u_{a}^{\min }, u_{a}^{\max }\right]$ is traveler's acceptable range for congestion pricing. However, Model P2 didn't consider the fairness. Due to the different occupation of road resources by different travel mode, the fairness after charge should be considered. In order to achieve a comparatively fairness, this paper takes the road resource occupation as fairness indication and transform traditional Model P2 with Gini coefficient and its defined constrains, so as to achieve the minimum total network cost and fairness at the same time. The upper Model P3 is:

$$
\begin{aligned}
& \text { (P3) } \min _{u} \mathrm{Z}(\boldsymbol{u}, \boldsymbol{x})=G(\cdot) \cdot\left\{\sum_{a \in B} x_{a}^{1} \cdot t_{a}^{1}\left(x_{a}^{1}\right)+\sum_{k} \sum_{r s} f_{r s, k}^{1} M_{r s, k}+\sum_{a \in A} x_{a}^{2} \cdot\left[t_{a}^{2}\left(x_{a}^{2}\right)+u_{a}\right]\right. \\
& \left.+\sum_{a \in A} x_{a}^{3} t_{a}^{2}\left(x_{a}^{3}\right)+\sum_{a \in B} x_{a}^{3} t_{a}^{1}\left(x_{a}^{3}\right)+\sum_{k} \sum_{p s} f_{p s, k}^{3}\left(T_{p}+M_{p s, k}\right)\right\} \\
& \text { s.t. } u_{a}^{\min } \leq u_{a}^{i} \leq u_{a}^{\max }, \forall i, a \\
& 0 \leq G_{1} \leq G(\cdot) \leq G_{2} \leq 1
\end{aligned}
$$

Wherein $\mathrm{G}(\cdot)$ is Gini coefficient; $\left[G_{1}, G_{2}\right] \subseteq[0,1]$ is its constraint. When $G(\cdot)=1$, Model P3 is the traditional Upper Model P2, so Model P3 is actually the extension of Model 2. Within the defined fairness range, in order to reach the minimum of objective function, the total network cost and Gini coefficient must be as low as possible thus to take both total cost and fairness into account.

The calculation of Gini coefficient evaluating the fairness of road resource occupation by travel mode resource is as follows ${ }^{[10]}$ :

(1) Determine road flow $\boldsymbol{x}^{i}$, route flow $\boldsymbol{f}^{i}$ and traffic demand $\boldsymbol{q}^{i}$ by travel modes;

(2) Calculate the time and space occupied by different travel modes according to road area and travel time per capita;

(3) Calculate the percentage of resource occupation and demographics and cumulative percentage of resource occupation and demographics;

(4) Match and draw Lorenz curve $L$ based on the above resource allocation data.

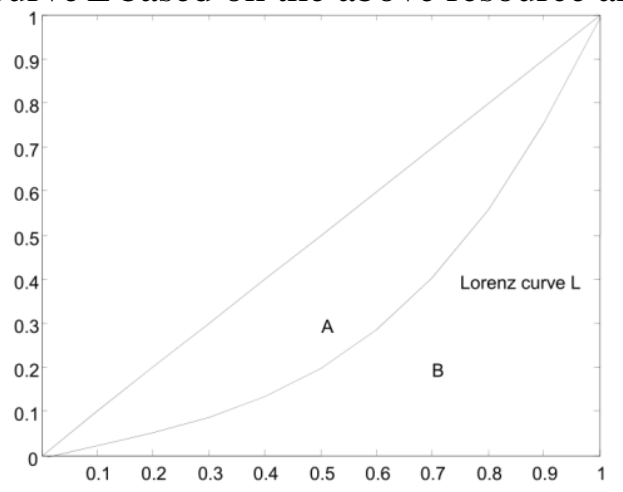

Figure 1 Lorenz curve

(5) Calculate the Gini coefficient $\mathrm{G}(\cdot)=\frac{S_{A}}{S_{A}+S_{B}}$, wherein $S_{A}$ and $S_{B}$ is the area of Region A and Region B in Figure 1.

\section{The two-layer model of Congestion Pricing for combined travel mode}

Above all, the two-layer model P4 of Congestion Pricing based on Gini coefficient for combined travel mode is: 
(P4) $\min _{u} \mathrm{Z}(\boldsymbol{u}, \boldsymbol{x})=G(\cdot) \cdot\left\{\sum_{a \in B} x_{a}^{1} \cdot t_{a}^{1}\left(x_{a}^{1}\right)+\sum_{k} \sum_{r s} f_{r s, k}^{1} M_{r s, k}+\sum_{a \in A} x_{a}^{2} \cdot\left[t_{a}^{2}\left(x_{a}^{2}\right)+u_{a}\right]\right.$

$\left.+\sum_{a \in A} x_{a}^{3} t_{a}^{2}\left(x_{a}^{3}\right)+\sum_{a \in B} x_{a}^{3} t_{a}^{1}\left(x_{a}^{3}\right)+\sum_{k} \sum_{p s} f_{p s, k}^{3}\left(T_{p}+M_{p s, k}\right)\right\}$

s.t. $u_{a}^{\min } \leq u_{a}^{i} \leq u_{a}^{\max }, \forall i, a$

$0 \leq G_{1} \leq G(\cdot) \leq G_{2} \leq 1$

Wherein $\boldsymbol{x}^{i}, \boldsymbol{f}^{i}$ is gained from lower model:

$$
\begin{aligned}
& \min Z\left(\boldsymbol{x}^{i}, \boldsymbol{f}^{i}, \boldsymbol{q}^{i}\right)=\sum_{i=1}^{3}\left[\frac{1}{\theta} \sum_{r s} \sum_{k} \int_{0}^{f_{r s, k}^{i}} \ln w d w-\left(\frac{1}{\theta}-\frac{1}{\beta}\right) \sum_{r s} \int_{0}^{q_{r s}^{i}} \ln w d w-\sum_{r s} h_{r s}^{i} q_{r s}^{i}\right] \\
& +\left[\sum_{a \in B} \int_{0}^{x_{a}^{1}} t_{a}^{1}(w) d w+\sum_{k} \sum_{r s} f_{r s, k}^{1} M_{r s, k}\right]+\sum_{a \in A} \int_{0}^{x_{a}^{2}} c_{a}^{2}\left(w, u_{a}\right) d w \\
& +\left[\sum_{a \in A} \int_{0}^{x_{a}^{3}} t_{a}^{2}(w) d w+\sum_{k} \sum_{r p} f_{r p, k}^{3} T_{p}+\sum_{a \in B} \int_{0}^{x_{a}^{3}} t_{a}^{1}(w) d w+\sum_{k} \sum_{p s} f_{p s, k}^{3} M_{p s, k}\right] \\
& \text { s.t. } \sum_{k} f_{r s, k}^{i}=q_{r s}^{i}, \forall r, s, i \\
& \sum_{i} q_{r s}^{i}=q_{r s}, \forall r, s \\
& x_{a}^{i}=\sum_{r s} \sum_{k} f_{r s, k}^{i} \delta_{a . k}^{r s}, \forall a, i \\
& f_{r s, k}^{i} \geq 0, x_{a}^{i} \geq 0, q_{r s}^{i} \geq 0, q_{r s} \geq 0, \forall r, s, i, k, a
\end{aligned}
$$

\section{Model calculation}

To solve Model P4, set congestion pricing scheme $\boldsymbol{u}=\left(\cdots, u_{a}^{i}, \cdots\right)$ and make the upper objective function the minimum. The steps are:

Step1. Determine the range of Gini coefficient based on fairness requirement;

Step2. Give the initial value of charging scheme $\boldsymbol{u}^{0}$, take iteration $n=0$;

Step3. Substitute $\boldsymbol{u}^{n}$ into the lower model to obtain the initial value of each travel mode $\boldsymbol{q}^{n}, \boldsymbol{x}^{n}, \boldsymbol{f}^{n}$;

Step4. Make out the road resource allocation table for each road travel mode according to the initial values $\boldsymbol{q}^{n}, \boldsymbol{x}^{n}, \boldsymbol{f}^{n}$, match the Lorenz curve $L^{n}(\cdot)$ and calculate the Gini coefficient $G^{n}(\cdot)$;

Step5. Substitute the Gini coefficient $G^{n}(\cdot)$ and the initial values $\boldsymbol{q}^{n}, \boldsymbol{x}^{n}, \boldsymbol{f}^{n}$ of different travel modes into the upper objective function, calculate the new road charging scheme $\boldsymbol{u}^{n+1}$;

Step6. Set iteration accuracy as $\varepsilon$, stop calculation when $\max \left|u_{a}^{i, n+1}-u_{a}^{i, n}\right| \leq \varepsilon(\forall a, i)$; otherwise, make $n=n+1$, ad skip to Step3.

\section{Example study}

Figure 1 shows a transportation network, consisting of 3 nodes 1,2,3. 1 OD corresponds to 4 sections, among which Section 4 is congestion charging section. Section 2 has parking spot and available bus transfer, dashed section 1, 3, 4 have buses.

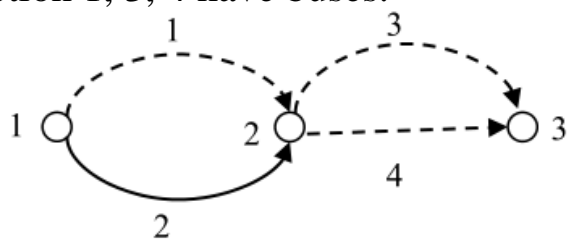

Fig.2 Schematic diagram of the road network 
The road travel time function $t_{a}^{i}(\cdot)$ takes BPR formula:

$$
t_{a}^{i}(\cdot)=t_{a, 0}^{i}\left[1+0.15\left(\frac{\gamma^{i} x_{a}^{i}}{C_{a}}\right)^{4}\right], i=1,2
$$

Wherein $\gamma^{i}(i=1,2)$ is the flow conversion coefficient, referring to the ration of PCU coefficient $\gamma_{P C U, k}$ to average passenger number. Set $\gamma^{1}=\frac{1}{20}, \gamma^{2}=1 ; t_{a, 0}^{i}(i=1,2)$ are the zero flow time of buses and cars on Section $a ; C_{a}$ is the actual capacity on Section $a$. The detailed values are shown in Table 1:

Table1 Table of value parameter

\begin{tabular}{cccc}
\hline Section $a$ & $\begin{array}{c}\text { Bus zero flow } \\
t_{a, 0}^{1}(\min )\end{array}$ & $\begin{array}{c}\text { Car zero flow } \\
t_{a, 0}^{2}(\min )\end{array}$ & $\begin{array}{c}\text { Capacity of Section a } \\
C_{a}(\mathrm{pcu} / \mathrm{min})\end{array}$ \\
\hline 1 & 6 & 4 & 350 \\
2 & - & 3 & 300 \\
3 & 9 & 6 & 350 \\
4 & 6 & 4 & 300 \\
\hline
\end{tabular}

Assume the fixed demand of OD to $(1,3)$ is $q_{13}=500 ; \theta=0.5, \beta=0.05$. The attractiveness of 3 travel modes are $h_{13}^{1}=10, h_{13}^{2}=5, h_{13}^{3}=9$ respectively; conversion value of bus fare and waiting time is $M_{13}=2, M_{23}=1$, parking charge $T_{2}=3$.

Table 2 Traffic flow before congestion charge

\begin{tabular}{cccccc}
\hline \multirow{2}{*}{ Section $a$} & $\begin{array}{c}\text { Bus flow } \\
x_{a}^{1}(\mathrm{p})\end{array}$ & $\begin{array}{c}\text { Car flow } \\
x_{a}^{2}(\mathrm{p})\end{array}$ & $\begin{array}{c}\text { Combined travel flow } \\
x_{a}^{3}(\mathrm{p})\end{array}$ & $\begin{array}{c}\text { Section flow } \\
x_{a}(\mathrm{PCU})\end{array}$ & Saturation $(\%)$ \\
\hline 1 & 102.9931 & 98.6370 & 51.6765 & 160.6128 & 53.5 \\
2 & 0 & 161.5379 & 85.1555 & 246.6934 & 70.5 \\
3 & 18.7886 & 72.3153 & 24.9617 & 99.15586 & 28.3 \\
4 & 84.2045 & 187.8596 & 111.8704 & 308.1505 & 102.7 \\
\hline
\end{tabular}

The flow $x_{a}$ in the table is the flow converted into the standard equivalent. Seen from Table 2, the saturation of Section 4 is $102.7 \%$, serious overcrowding. Consider to charge at Section 4 to change traveler's route to alleviate the congestion on Section 4.

Set the road area per capita occupied buses and cars as $1.25 \mathrm{~m}^{2} / \mathrm{p}, 20 \mathrm{~m}^{2} / \mathrm{p}$. the urban road resources allocation before congestion pricing OD to $(1,3)$ is seen in Table 3 :

Table 3 Urban road resources allocation before congestion pricing

\begin{tabular}{cccccccc}
\hline $\begin{array}{c}\text { Travel } \\
\text { mode }\end{array}$ & $\begin{array}{c}\text { Traveler } \\
(\mathrm{p})\end{array}$ & $\begin{array}{c}\text { Travel } \\
\text { time } \\
(\mathrm{min})\end{array}$ & $\begin{array}{c}\text { Time\& } \\
\text { Space } \\
\text { resource } \\
\left(\mathrm{m}^{2} \cdot \min \right)\end{array}$ & $\begin{array}{c}\text { Resource } \\
\text { percentage } \\
(\%)\end{array}$ & $\begin{array}{c}\text { Resource } \\
\text { Cumulative } \\
(\%)\end{array}$ & $\begin{array}{c}\text { Demographics } \\
\text { percentage } \\
(\%)\end{array}$ & $\begin{array}{c}\text { Demographics } \\
\text { Cumulative } \\
(\%)\end{array}$ \\
\hline $\begin{array}{c}\text { Bus } \\
\begin{array}{c}\text { Combined } \\
\text { travel }\end{array}\end{array}$ & 102.993 & 21 & 1615.4 & 3 & 3 & 20.6 & 20.6 \\
Car & 260.175 & 17 & 41719 & 77.7 & 100 & 27.4 & 48 \\
\hline
\end{tabular}

According to data in Table 3, match and draw Lorenz curve and calculate the Gini coefficient as 0.2993. According to the relevant provisions of United Nations organizations: Gini coefficient $G(\cdot)<0.2$ indicates absolute fairness; $0.2 \leq G(\cdot)<0.3$ fairness; $0.3 \leq G(\cdot)<0.4$ relatively fairness; $0.4 \leq G(\cdot)<0.5$ indicates relatively unfairness; $G(\cdot) \geq 0.5$ indicates extremely unfairness. Therefore, the road resource allocation of each travel mode is relatively unfairness in SUE state.

Set congestion pricing range ${ }^{[3,10]}$, solve Model P4. In order to make the congestion pricing to be relatively fair, make the Gini coefficient constraint range $[0.3,0.4)$. Calculate and get $u_{4}^{2}=3$ using 
matlab program. That is, when charging 3 time unit of cost to cars on Section 4, the upper model objective function achieve the minimum value as 2455.1 and Gini coefficient as 0.3878 .

Then verify whether the charge alleviate traffic congestion. The flow on sections after the charge is as shown in Table 4:

Table 4 Traffic flow after congestion charge

\begin{tabular}{cccccc}
\hline \multirow{2}{*}{ Section $a$} & $\begin{array}{c}\text { Bus flow } \\
x_{a}^{1}(\mathrm{p})\end{array}$ & $\begin{array}{c}\text { Car flow } \\
x_{a}^{2}(\mathrm{p})\end{array}$ & $\begin{array}{c}\text { Combined travel flow } \\
x_{a}^{3}(\mathrm{p})\end{array}$ & $\begin{array}{c}\text { Section flow } \\
x_{a}(\mathrm{PCU})\end{array}$ & Saturation $(\%)$ \\
\hline 1 & 128.6687 & 75.8004 & 64.5565 & 153.2238 & 51.1 \\
2 & 0 & 124.6741 & 106.3004 & 230.9745 & 66 \\
3 & 23.4725 & 124.5055 & 31.1687 & 158.0215 & 45.1 \\
4 & 105.1962 & 75.9689 & 139.6882 & 226.1767 & 75.4 \\
\hline
\end{tabular}

Seen from Table 4, saturation in Section 1, 2, 4 decrease, and congestion is eased, while saturation on Sections 3 increased slightly. After charging, the car flow on Section 4 decreases, less people choose to drive office and more people turn to high-capacity buses and combined travel, thus to alleviate the congestion.

The relationship between congestion charge and Gini coefficient is as shown in Figure 3.

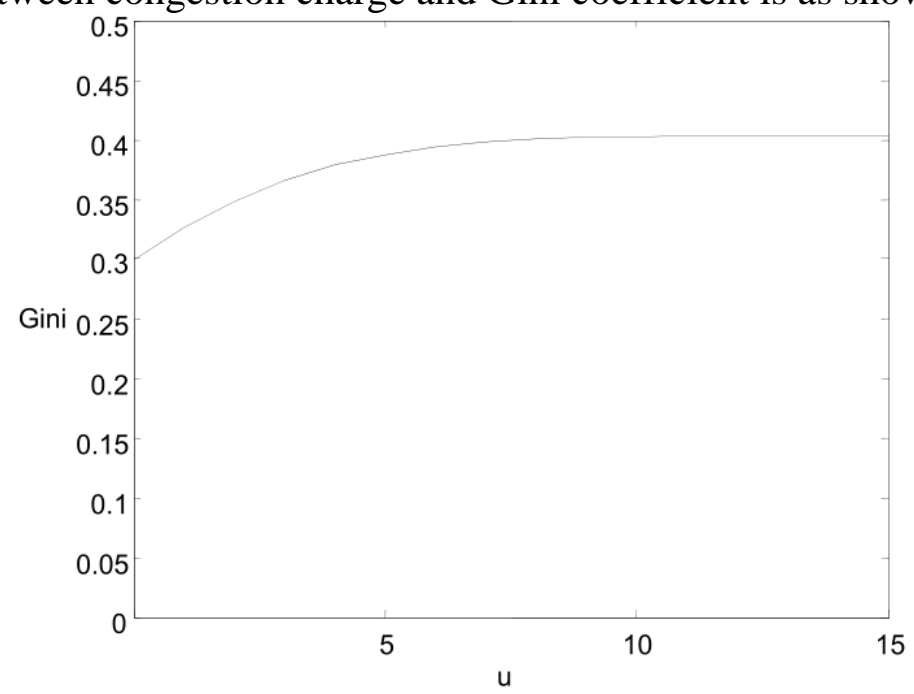

Fig.3 Relationship between congestion charge and Gini coefficient

Figure 3 shows that, with the increase of congestion charge, Gini coefficient increase and unfairness increases correspondingly. This is because after charging, part of driving the whole travel turn to bus travel or combined travel that occupies less road resource. That is to say people using travel modes with less road resource increase, so does the unfairness. When the congestion charge is 8 , the Gini coefficient is 0.4001 and exceeds the warning value. Therefore, when applying the model for congestion pricing design, the administrator must select the appropriate charge range and Gini coefficient constraint according to financial objective and fairness requirement.

\section{Conclusion}

In real life, bus travel, driving the whole travel and combined travel are the most common transportation modes. This paper studies the congestion pricing by combined travel and build an integrated decision-making two-layer model of park-and-ride and congestion pricing. Taking into fairness into account, with road resource occupation by different modes as fairness indication, the model transforms traditional upper model with Gini coefficient and its constraint. The example study has verified that the implementation of parking charge and congestion pricing alleviate the road congestion with fairness. This paper proposed the lower model using SUE Model of fixed demand, which can be also of elastic demand, and use Gini coefficient to extend the traditional model. 


\section{References}

[1] Meng Meng, Shao Chunfu, Zeng Jingjing, Xu Qingzhu. Combined Travel Mode and Algorithm under Park-and-Ride [J]. Jilin University Journal, 2013, 43 (6): 1465-1471.

[2] Qin Huanmei, Guan Hongzhi, Pan Xiaosong. Park-and-Transfer Demand based on Random Continuous Equilibrium Model [J]. Jilin University Journal, 2012, 42 (2): 321-326.

[3] Huang Haijun, Li Zhichun. The Mixed Equilibrium Model and Algorithm of Combined Travel Mode [J]. Systems Science and Mathematics, 2006, 26 (3): 352-361.

[4] Li Zhichun, Combined Travel Mode under Elastic Demand Model and Algorithm [J]. Chinese Journal of Highway, 2005,18 (3): 94-98.

[5] Li Honglian. Research on Urban Multimodal Transportation Allocation under Available Transfer [D]. Beijing, Beijing Jiaotong University Transportation Institute, 2011.

[6] Shi Feng, Chen Yan, Li Hengxin, Deng Lianbo. Research on Comprehensive Optimization of 1-Level Congested Roads and Parking Facilities Charge [J]. Journal of Transportation Systems Engineering and Information Technology, 2009,9 (1): 74-79.

[7] Li Hengxin, Hou Guirong, Huang Zhengcheng. Research on Network Congestion Road Pricing Model and Algorithm of Combined Travel under Elastic Demand [J]. Highway Engineering, 2009, 34 (1): 46-48.

[8] Wang Jian, Sun Guanglin. Optimization of Road-based Congestion Charge and Parking Charge [J]. Journal of Transportation Systems Engineering and Information Technology, 2010,10 (3): 24-28.

[9] Wei Zengxin, Zhao Qiumei, Gao Suluan, Kuo Chaohui. Optimization of Congestion Charge and Parking Charge on Congested Road [J]. Journal of Transportation Information and Safety, 2012, 30 (3): 1-5.

[10] Juan Zhicai, Luo Qingyu, Fu Zhongning, Gu Hongfei. Impact of Congestion Charge on Urban Road Resource Allocation Fairness [J]. Journal of Transportation Systems Engineering and Information Technology, 2008, 8 (1): 74-79.

[11] Wu Maolin, Cao Kai. Quantitative Evaluation of Transportation Equity based on Gini Coefficient [J]. Technology \& Economy in Areas of Communications, 2011 (1): 85-89.

[12] Zhang Xiaoning, Cao Jin. Analysis on Social Equity of Congestion Charge [J]. Tongji University Journal, 2010, 38 (11): 1605-1609.

[13] Shi Jing, Yang Lang, Huang Qian, Ying Xiwen. The Evaluation Angle and Model Description on Transportation Equity [J]. Journal of Railway Engineering Society, 2009 (1): 97-101.

[14] Li Yishun, Xu Min, Lu Kai. Combined Measures for Regional Congestion Charge and Park-and-Ride [J]. Journal of South China University of Technology, 2011, 39 (10): 100-104.

[15] Luo Chaohui, Wei Zengxin, Chen, Jinlai. Research on Equilibrium Travel Decision Model of Urban Multimodal Random Travelers [J].Mathematics in Practice and Theory, 2012, 42 (5): 98-106. 